\title{
B-scan opthalmic Ultrasonography: a review corroborated with echograms
}

\author{
Uduma Felix Uduma ${ }^{1 *}$, Akpan Sylvia Iquo-Abasi ${ }^{2}$ and Njeze Ngozi Rosemary ${ }^{3}$ \\ ${ }^{1}$ Department of Radiology, Faculty of Clinical Sciences, University of Uyo, Uyo, Nigeria \\ ${ }^{2}$ Department of Ophthalmology, Faculty of Clinical Sciences, University of Uyo, Uyo, Nigeria \\ ${ }^{3}$ Department of Radiation Medicine, Faculty of Medical Sciences, University of Nigeria, Enugu, Nigeria
}

\begin{abstract}
There are many investigative methods employed by Ophthalmologists to establish diseases of the eyes. An example is ophthalmoscopy. But this cannot explicitly evaluate posterior eye segment in cases of opaque vitreous or vitreous haemorrhages. The resort to B-scan ophthalmic ultrasonography seamlessly aids in non-invasive, cheap, real-time and reproducible diagnosis of ocular pathologies. Some of these ocular pathologies with supportive echograms observed in the course of our practice are reviewed in this study.
\end{abstract}

\section{Teaching points}

1. B-scan ocular ultrasonography is a cheap, readily available and reproducible means of evaluating lesions of the eyes using high frequency transducers.

2. B-scan ocular ultrasonography is a good substitute to ophthalmoscopy in evaluation of posterior segment of the eye in dense vitreous and vitreous haemorrhages.

3. B-scan ocular ultrasongraphy can readily detect ocular pathologies and this can further be improved with augmentation with dynamic ocular scan.

4. Definitive detection of the various types of ocular detachments is the beauty of B-scan ocular ultrasonography.

5. Contraindication for B-scan ocular ultrasonography is globe rupture.

\section{Introduction}

Ocular B-mode ultrasonography (USS) is an essential adjuvant for evaluation of ocular diseases. Its use in ophthalmic evaluation dates to $1950 \mathrm{~s}[1,2]$. This is not unconnected with the anatomic accessibility of the eye. The cystic nature of the eye also made it a good candidate for sonographic evaluation, a modality that is based on high frequency sound waves. But the beauty of ocular ultrasonography is its role as a substitute to ophthalmoscopy or as an ancillary to some opthalmoscopic limitations. For example, it gives instantaneous information on lesions in eyes with opaque ocular media $[1,3,4]$. This opaque medium would ordinarily have conferred some hindrances to adequate ophthalmoscopic visualization of the posterior segment of the globe. Direct visualization of the retro -bulbar pathologic lesions with ophthalmoscope is also not possible even with clear media [1]. This therefore calls for a substitutive imaging modality like B-scan ocular ultrasonography that will readily characterize most ocular lesions. These lesions range from lens pathology to posterior eye segment pathologies.
Ocular B-scan produces a two-dimensional cross-sectional display of the globe and the orbit $[1,3]$. It is a cheap, non-invasive, real time and quick imaging modality with good spatial resolution. It could also be performed at the patient's bedside. Moreover, ocular B-scan can be done simultaneously with eye movement and this is referred as dynamic study. This dynamic study is incorporated in distinguishing the different types of ocular detachments as well as mobility of intravitreous echoes $[1,3]$ One could also have used computed tomography (CT) and magnetic resonance imaging (MRI) in many ocular and orbital conditions. But they cannot scan in real time and they have limited role in the evaluation of the vitreous, retina and choroid detachments $[1,3]$. The above pitfalls justify our choice of B-scan ultrasonography in evaluations of ocular pathologies.

\section{Aim}

To highlight the usefulness of B-scan ultrasonography in elucidation of ocular pathologies.

\section{Evaluations of ocular pathologies based on affected structures}

Pathologies of the anterior eye segment: Moving in anterior posterior direction, the anterior eye segment consists of cornea, anterior chamber, iris, ciliary body, posterior chamber and lens. These are structures that rarely require USS, as they are readily assessed by clinical evaluations, ophthalmoscopy, slit-lamp examination, and ultrasound bio microscopy. The later uses frequencies as much as 35- 50 $\mathrm{MHz}$ to evaluate abnormalities like ocular trauma, foreign bodies and opaque elements in the anterior segment [2,3,5-8]. Notwithstanding

${ }^{\star}$ Correspondence to: Felix Uduma U, Department of Radiology, Faculty of Clinical Sciences, University of Uyo, Uyo, Nigeria, E-Mail: felixuduma@yahoo.com

Key words: ocular ultrasonography, b-scan, ophthalmoscopy, vitreous, retina, detachments

Received: April 15, 2019; Accepted: April 25, 2019; Published: April 29, 2019 
the core utility of USS in posterior eye segment evaluations, it has also been used to localize intra-ocular foreign body (IOFB) in the anterior chamber thereby defining the size, shape and nature of the IOFB [1]. On USS, small metallic IOFB in the anterior eye segment will appear as a markedly echogenic short linearity that cast comet tail artefact.

Lens pathology: Pathologies of the lens of the eyes are known to cause visual impairment or blindness in millions of people. The major lens pathology is cataract which is a degenerative condition. Cataract is defined as any opacity of the lens that results in significant variations in the refractive index of the lens over distances like the wavelength of transmitted light. World Health Organization (WHO) documented 18 million people to be blind from cataract. Common causes of cataract include long-term exposure to the sun's ultraviolet light, aging, inherited disorders, diabetes mellitus and trauma $[9,10]$.

Cataract could be congenital, juvenile, pre-senile or senile. The latter is the commonest. Cataracts could also be classified as traumatic, immature, mature and hyper mature cataracts. On ophthalmoscopy, cataract is seen as a white reflection with an opaque lens called leukocoria. B-scan is important in cataracts as it evaluates the posterior eye segment which may be difficult with ophthalmoscopy. Such preoperative B-scan is to rule out possible contraindications to cataract extractions such as retinal detachment or tumours. On B-scan, features of cataracts range from completely opaque hyperechoic lens cortex, intra-lenticular echoes, reflective material beneath the lens capsule, thick hyperechoic posterior capsule of the lens and combined thick hyperechoic anterior and posterior lens capsules. In immature cataracts, scattered opacities are separated by clear zones whereas in mature cataract, the thick echogenicity on the posterior capsule of the lens is a continuous plaque [3].

Lens dislocation: Lens dislocation could be traumatic, spontaneous or it could originate from hereditary or connective tissue diseases. Examples of the last aetiologies are Marfan's syndrome, congenital aniridia, congenital glaucoma, Ehlers -Danlos syndrome, homocystinuria and molybdenum cofactor deficiency [11]. The commonest cause is trauma. Here, blunt force in anterior- posterior direction leads to globe equatorial expansion leading to zonular fibres disruption and lens dislocation [12-14]. Spontaneous lens dislocation can result from myopia. Partial zonular dialysis may lead to partial lens dislocation (subluxation) and complete zonular rupture leads to complete lens dislocation (ectopia lentis) [11]. In partial dislocation, the crystalline lens remains partially attached to one ciliary body and has an oblique axis. In partial lens dislocation, scan is done in different positions as the globulous lens might be in normal position when patient lies supine. In a complete dislocation, the lens sinks into the vitreous body in a dependent position, lying over the retina. It moves during dynamic examination, simulating floating lens sign seen in CT. The delayed complications of lens dislocation include secondary or phacolytic glaucoma, delayed retinal detachment, allergic uveitis, proliferative vitreoretinopathy, cataract and vision loss [11]. The secondary glaucoma resulting from lens dislocation is caused by a synechia between the iris and the lens, causing the iris to curve forward, and termed iris bombe. This prevents the flow of aqueous humour from the posterior to the anterior chamber. Treatment of choice for lens dislocation is surgical repair, repositioning, explanting, or exchanging the displaced intraocular lens (Figures 1 and 2) [13].

\section{Pathologies of the posterior eye segment}

Vitreous pathologies: Vitreous pathologies are multifarous and they range from vitreous haemorrhages, vitreitis, asteroid hyalosis,

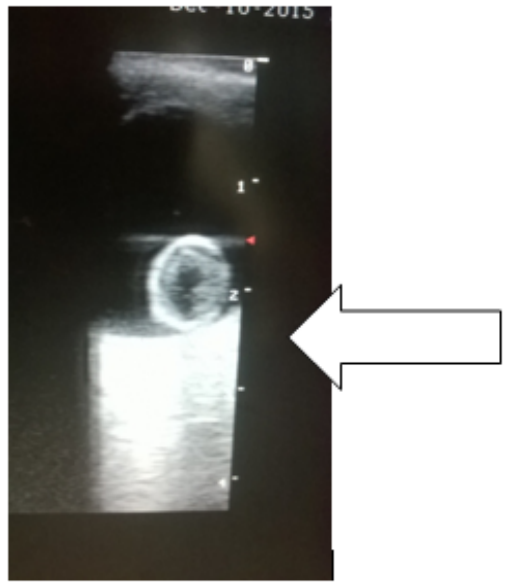

Figure 1. Coned section of an ocular echogram showing the globulous crystalline lens [white arrow] that has migrated to the posterior vitreous (a dependent position) and abutting the papilla and adjacent retina of the right eye of a patient (Complete dislocation of the lens of the right eye)

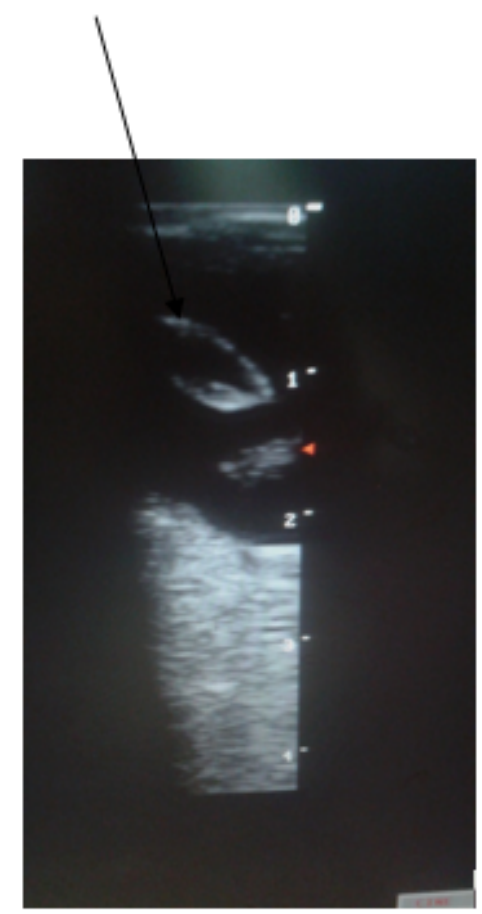

Figure 2. Coned section of an ocular echogram of the right eye of another patient. The ovoid echogenic rim lens is pendulous and obliquely orientated in the anterior vitreous [Black oblique arrow]. One pole of the lens is still attached to one of the ciliary bodies of the right eye (Partial dislocation of the lens of the right eye)

persistent hyperplastic primary vitreous, coloboma, vitreous degeneration, posterior vitreous detachment, vitreoschisis, and retrohyaloid haemorrhage.

\section{Vitreous floaters, vitreous haemorrhages, vitreous degeneration, vitreitis and asteroid hyalosis}

The vitreous cavity is filled with transparent vitreous fluid or gel. The vitreous fluid is an acellular viscous fluid with $99 \%$ water content and the rest consists of type 2 collagen fibrils and hyaluronic acid [3]. However, due to ageing, liquefaction of the vitreous called syneresis occurs. This will lead to ultrasonographic detection of few low-amplitude punctate and linear mobile echoes floating within the 
vitreous chamber, often referred to as "floaters" [3,15,16]. Bilateral multiple hyper-reflective vitreous floaters that show after-movements on a dynamic scan in an elderly without known instigating causes of vitreous haemorrhage, may suggest vitreous degeneration. In vitreous degeneration, the liquefied vitreous contains cholesterol crystals as floaters, also known as synchysis scintillans [4].

Vitreous haemorrhages are caused by trauma, proliferative vascular retinopathies, coagulopathies, age related macular degenerations, retinal breaks, retinal microaneurysms, posterior vitreous detachment (PVD) and neoplasia. On USS, they appear as reflective intra-gel mobile dot echoes that are dependent and freely moves with dynamic scan. Acute bleed will appear as echo lucent or low reflective echoes. These haemorrhages may clear in 2-4 weeks or become chronic. When haemorrhages become chronic, the dotlike echoes get organized to form fibrinous vitreous membranes of varying reflectivity and devoid of anatomic attachment $[3,8,16-18]$

Vitreitis may mimic vitreous haemorrhages but the vitreous echoes will be less mobile and may have associated membranous echoes. But other supportive USS findings of ocular inflammation will be visible, and they include retinochoroidal thickening, exudative retinal detachment, optic disc elevation or Tenon's space widening. Asteroid hyalosis is an asymptomatic degenerative condition resulting from calcium soaps accumulation on vitreous fibrils in the eye [17]. It is usually unilateral, and the cause is unknown, but it is common in diabetes mellitus. On USS, the vitreous cavity shows a variable number of discrete mobile bright echoes (reminiscent of the particles in a snow globe) with a classical space between the particles and posterior globe wall (Figure 3) $[3,8,16,17]$.

Posterior vitreous detachment (PVD): PVD is an-age related phenomenon whereby the posterior vitreous capsule or hyaloid detaches from the underlying retina [3]. It is also called hyaloids detachment and it can be an incidental finding or pathological. But it is seen in two-thirds of people older than 65 years [19]. PVD can be partial or complete. It can also be focal or broad, single or multiple and its reflectivity depends on aetiology [17]. On USS especially on dynamic scan, it characteristically shows as an undulating membrane that moves freely and should swirl away from the region of the optic disc in cases of complete posterior vitreous detachment [3]. Note that the mobility of PVD is more than that of retinal detachment (Figure 4).

Retro-hyaloid haemorrhage: It is also called sub-hyaloid haematoma or posterior hyphema. Vitreoretinal adhesions can cause retinal tears or avulsion of a peripheral blood vessel, resulting in vitreous and retro-hyaloid haemorrhage [3]. It can also be due to vitreoschisis which is splitting of the posterior cortical vitreous, common in diabetic retinopathy [17]. On USS, retro-hyaloid haemorrhages appear as multiple low reflective dot echoes posterior to PVD which may demonstrate layering of echoes with a straight surface but moves with head movement [17].

Persistent hyperplastic primary vitreous: This is also called persistence of fetal vasculature. Persistent hyperplastic primary vitreous (PHPV) is a congenital developmental anomaly arising from failure of regression of embryological primary vitreous and hyaloid vasculature and presenting as leukocoria $[3,20]$. It is idiopathic, typically unilateral but $10 \%$ are bilateral. USS shows as microphthalmia, retrolental high reflective thick fibrovascular linear membrane extending from the lens to the optic disc, thin lens with irregular, congenital cataractous posterior capsule and peri-papillary tractional retinal detachment $[3,4,17,21,22]$. The pattern of retinal detachment in PHPV is usually

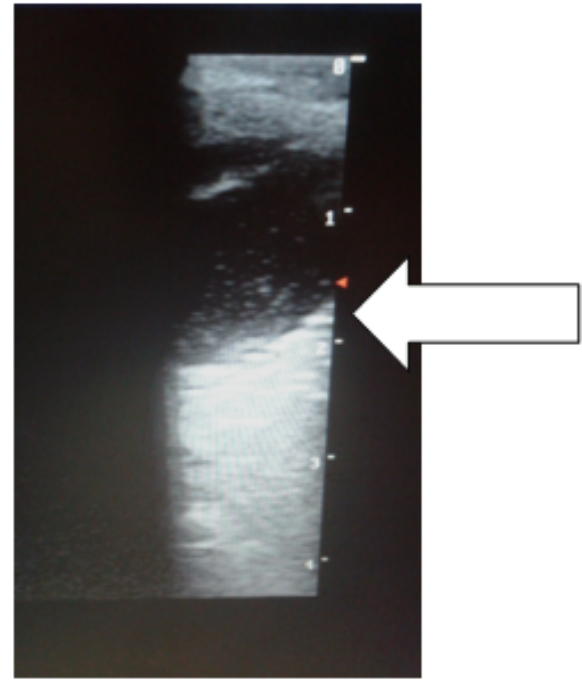

Figure 3. Coned view of the left eye of a patient showing floating intra-gel dot echoes of vitreous haemorrhages [White horizontal arrow]

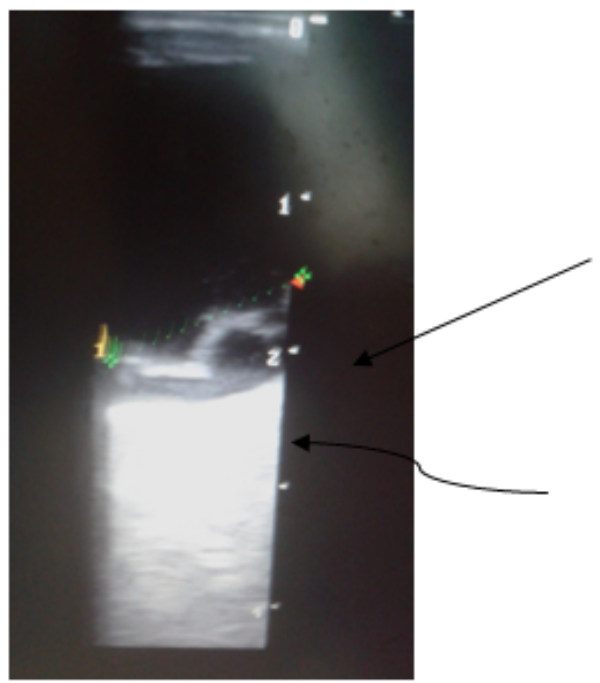

Figure 4. Coned section of an ocular echogram of the left eye of a patient. This shows an echogenic undulating thick-walled membrane [black oblique arrow] anterior to the retina. Note associated retro-hyaloid haematoma that is seen as echogenic debris beneath the membrane [curved black arrow]

funnel shaped suggestive of total retinal detachment. A persistent hyaloid artery may also be present in the centre of retrolental band detected with Doppler scan.

\section{Pathologies of retina}

Retinal pathologies include retinal detachment, retinal tear, subretinal haemorrhages, retinal cysts.

\section{Retinal detachment}

Retinal detachment (RD) is the detachment of the inner sensory layer of the retina from the outer pigmented layer called retinal pigmented epithelium $[3,23]$. Basically, $\mathrm{RD}$ is categorized as rhegmatogenous $\mathrm{RD}$, tractional $\mathrm{RD}$, and exudative $\mathrm{RD}$. $\mathrm{RD}$ could also be total, partial, or focal, depending on its extension. The classical USS features of total $\mathrm{RD}$ is a "V" shape echogenic lesion in the vitreous cavity with anterior attachment to the ora serrata and posterior 
attachment to the optic nerve head [3,17]. On the other hand, partial $\mathrm{RD}$ will show as an intra-vitreal linear echogenic membrane extending to the optic nerve head, but not across it $[3,23]$. The leaflets of the membranes in acute RD are thinner and more mobile than in chronic $\mathrm{RD}$. Thus, Chronic RD is often seen as a rigid "triangle sign" [3,15,18,24]. Sometimes, retinal cyst can form in chronic $\mathrm{RD}$. The retina can have an open or tightly closed funnel configuration in cases of complete RD seen in conditions like proliferative vitreoretinopathy, retinopathy of prematurity, PHPV and retinoblastoma. Exudative and tractional RD are often associated with subretinal haemorrhages (Figure 5).

\section{Sub-retinal haemorrhages}

Causes of sub-retinal haemorrhages are trauma, age related macular degeneration, intra-papillary haemorrhages, diabetes mellitus, retinal tear, $\mathrm{RD}$ and ocular tumours $[17,25,26]$. On USS, sub-retinal haemorrhages present as echogenic debris beneath an elevated retina. This debris has variable reflectivity due to clotted or lysed blood. The debris might be mobile on dynamic scan or having a shifting contour. It tends to change contour and reduction in height with time due to resolution or breakthrough vitreous haemorrhage [17].

\section{Retinoblastoma}

Retinoblastoma is the commonest childhood malignant ocular tumour. It is seen in 1 in 17,000 live births with mean age at presentation of 2 years for unilateral forms and one year in bilateral forms [27]. The tumour arises from the retina and could be exophytic (subretinal) or endophytic (growing towards the vitreous cavity) [17]. On USS, it could present as a single or multiple solid mass with irregular high internal reflectivity due to presence of calcium $[17,28]$. Internal calcification is very characteristic as it seen in up to $90 \%$ of cases (Figure 6).

\section{Choroidal pathologies}

\section{Choroidal detachment}

Choroidal detachment is caused by trauma, hypotony, corneal ulcers, intra-ocular pressure lowering agents, ocular surgeries and RD. Choroidal detachment can also cause $\mathrm{RD}$ [29]. In choroidal detachment fluid accumulates in the supra-choroidal space and this fluid can be

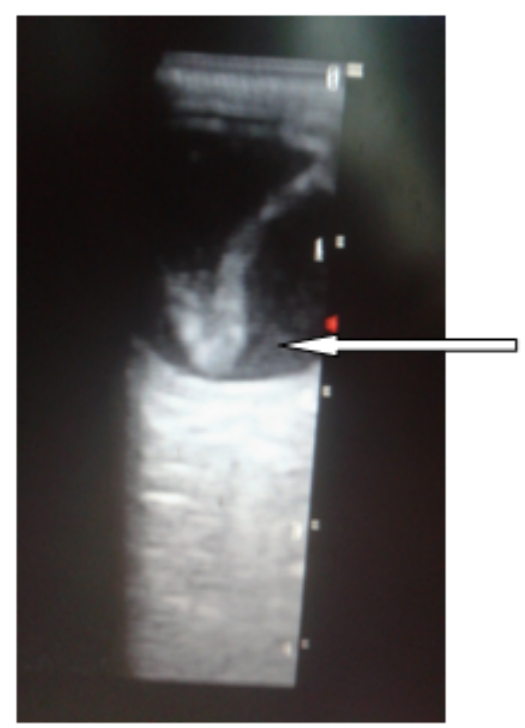

Figure 5. Coned view of an echogram of the left eye of a patient. This shows a V-shaped echogenic band [white thin arrow] in the posterior vitreous. This is anchored posteriorly to the papilla and anteriorly to the ora serrata

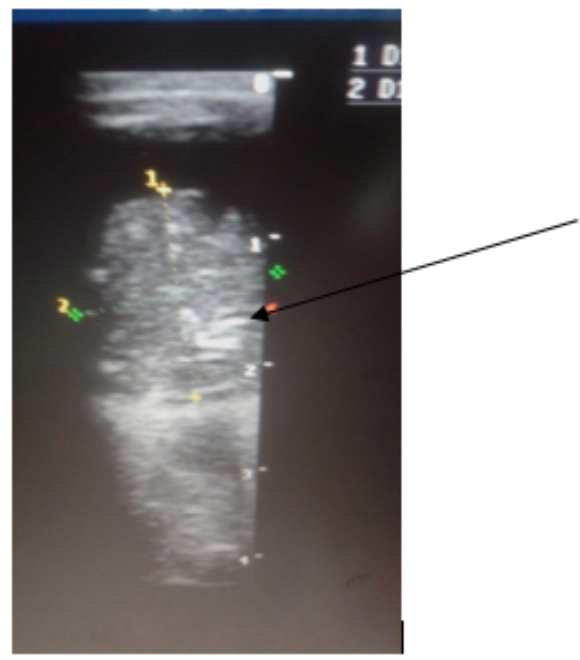

Figure 6. A coned view of a right eye echogram of a child showing an intra-vitreous solid heterogenous mass arising from the retina [black oblique arrow]. Note associated multiple internal punctuate calcifications (Retinoblastoma)

echo lucent or echo rich. On USS, choroidal detachment presents as a dome shaped thick membrane in the periphery and it does not extend beyond the equator as its limited by the vortex vein exit inferiorly [17].

\section{Other ocular morphologies}

\section{Alterations in size and shape of the ocular globe}

The normal antero-posterior diameter of the ocular globe is approximately 22 to $25 \mathrm{~mm}$ in adolescents and adults. In patients with long-term myopia, the antero-posterior axis of the globe is lengthened. The globe sometimes develops a thin wall, often manifesting as a pear-shaped sacculation of the posterior pole. This is referred to as a posterior staphyloma [3].

\section{Phithiasis bulbi}

Phithiasis bulbi is the terminal stage of many ocular lesions and this include trauma, infection and surgery. Here, the eye is redundant, micropthalmic, blind, non-functioning, with extensive calcifications. The affected eye has crenated, shrunken globe with extensive calcification and loss of the normal shape. Cataracts, partial lens dislocation, vitreous fibro-vascular membrane and retinal detachment may also be seen $[3,4,30]$.

\section{Conclusion}

Ocular B-scan is a cheap, convenient and readily available tool for diagnosis of diseases of the eyes. This is most valuable in diseases of posterior eye segment especially in opaque vitreous or vitreous haemorrhage.

\section{References}

1. Bengal SV, Bhandari AJ, Siddhiqui F (2016) Pattern of ocular pathologies diagnosed with B-scan ultrasonography in a hospital in rural India. Niger J Ophthalmol 24: 71-75.

2. Brunell KS (2014) Opthalmic ultrasonography. J Diagn Med Sonography 30:136-142.

3. De La Hoz P, Torramilans LA, Pozuelo SO, Anguera BA, Esmerado AC, et al. (2016) Ocular ultrasound focussed on the posterior eye segment : What Radiologists should know. Insight Imag 7: 351-364. [Crossref]

4. Aironi VD, Gandage SG (2009) Pictoorial essay: B-scan ultrasonography in ocular abnormalities. Indian J Radiol Imaging 19: 109-115. [Crossref]

5. Andreoli MT,Yiu G, Hart L,Andreoli CM (2014) B-scan ultrasonography following open globe repair. Eye 28: 381-385. [Crossref] 
6. Agrawal R, Ahirwal S (2015) A study of role of B scan ultrasound in posterior segment pathology of eye. Int J Med Res Rev 3: 969-974.

7. Silverman RH (2009) High-resolution ultrasound imaging of the eye: a review. Clin Experiment Ophthalmol 37: 54-67.

8. Bedi DG, Gombos DS, Ng CS, Singh S (2006) Sonography of the eye. Am J Roentgenol 187:10610-1072. [Crossref]

9. Wormstone M, Wride M A (2011) The ocular lens:a classic model for development, physiology and disease. Philos Trans R Soc Lond B Biol Sci 366: 1190-1192. [Crossref]

10. Michael R, Bron A (2011) The ageing lens and cataract : A model of normal and pathological ageing. Philos Trans R Soc Lond B Biol 366: 1278-1292.

11. Vyas S, Krishna S, Kumar A, Khandelwal N (2015) 'Floating lens sign in traumatic lens dislocations'. Middle East Afr J Ophthalmol 22: 129-130. [Crossref]

12. Mathur SK, Grodinsky D (2003) Dislocation of the lenses. N Engl J Med 35: e22.

13. Lee S, Hayward A, Bellamkonda VR (2015) Traumatic lens dislocation. Int J Emerg Med 8: 16.

14. Fischel JD, Wishart MS (1995) Spontaneous complete dislocation of the lens in Pseudoexfoliation syndrome. . J Catar Refract Surg 7: 31-33.

15. Roque PJ, Hatch N, Barr L, Wu TS (2014) Bedside ocular ultrasound. Crit Care Clin 30: 227-241. [Crossref]

16. Munk PL, Vellet AD, Levin M, Lin DT, Collyer RT (1991) Sonography of the eye. AJR Am J Roentgenol 157: 1079-1086.

17. Bhende M, Gopal S, Gogi A, Sharma T, Gopal L, et al. (2006) The Sankara Nethralaya Atlas of Opthalmic ultrasound,New Delhi; Jaypee Brothers Medical Publishing 1st edn pp 27-51.

18. Fielding JA (2004) The assessment of ocular injury by ultrasound. Clin Radiol 59: 301-312. [Crossref]
19. Sebag J (2018) Posterior vitreous detachment. Ophthalmolgy 125: 1384-1385. [Crossref]

20. Bezuidenhout AF (2016) Persistent hyperplastic primary vitreous? The martiniglass sign. S Afr J Rad 2014 18: 597.

21. Tartarella MB, Takahagi RU, Braga AP, Fortes Filho JB (2013) Persistent fetal vasculature: Ocular features, management of cataract and outcomes. Arq Bras Oftalmol 76: $185-188$ [Crossref]

22. Jain TP (2009) Bilateral persistent hyperplastic primary vitreous. Indian J Ophthalmol 57: 53-54. [Crossref]

23. Silva CT, Brockley CR, Crum A, Mandelstan SA (2011) Pediatric ocular sonography Semin Ultrasound CT MR 32: 14-27.

24. Dessì G, Lahuerta EF, Puce FG, Mendoza LHR, Stefanini T, et al. (2014) Role of B-scan ocular ultrasound as an adjuvant for the clinical assessment of eyeball diseases: A pictorial essay. J Ultrasound 18: 265-277 [Crossref]

25. Moon IH, Lee SC, Kim M (2018) Intra-papillary haemorrhage with concurrent peripaillary and vitreous haemorrhage in two healthy young patients. BMC Ophthamology 18: 172.

26. Maguire SA, Watts PO, Shaw AD, Holden S, Taylor RH, et al. (2013) Retinal haemorrhages and related findings in abusive and non-abusive head trauma: a systematic review. Eye 27: 28-36. [Crossref]

27. de Graaf P, Goricke S, Rodjan F, Galluzzi P, Maeder P, et al. (2012) Guidelines for imaging retinoblastoma: imaging priniciples and MR standardization. Pediatr Radiol 42: 2-14.

28. Razek AAKA, Elkhamary S (2014) MRI of retinoblastoma. Br J Radiol 84: 1005 [Crossref]

29. Seoko M (2016) Choroid detachment, a rare cause of vision loss diagnosed by point-ofcare ultrasound. J Emerg Med 52: 4. [Crossref]

30. Kubal WS (2016) Imaging of orbital trauma. Radiographics 28: 1729-1739. [Crossref]

Copyright: (C2019 Felix Uduma U. This is an open-access article distributed under the terms of the Creative Commons Attribution License, which permits unrestricted use, distribution, and reproduction in any medium, provided the original author and source are credited. 